Pacific Journal of Mathematics

DERIVATIVES OF THE HARMONIC MEASURES IN 


\title{
DERIVATIVES OF THE HARMONIC MEASURES IN MULTIPLY-CONNECTED DOMAINS
}

\author{
Michael MaschleR
}

\section{Introduction. Basic definitions and some known results.}

The Geometric Function Theory encounters serious difficulties when dealing with multilply-connected domains, due to the fact that no domain function is known in an explicit form for a domain of connectivity greater than two. It is for this reason that one at least tries to find properties of the domain functions in terms of geometric characteristics of their domains.

In this paper we search for information on domain functions and certain families of functions, defined for $p$-connected domains, that can be deduced form properties of functions defined in symmetric domains of connectivity $2(p-1)$. We also extend the results to infinitely-connected domains.

Let $\Delta$ be a domain in the $z$-plane, and let $L^{2}(\Delta)$ be the class of functions which are regular and square integrable in $\Delta$. Let $l^{2}(\Delta)$ be its subclass consisting of those functions which have a single-valued integral in $\Delta$. Both classes form separable Hilbert Spaces $\left[L^{2}(\Delta)\right]$ and $\left[l^{2}(\Delta)\right]$ under the scalar multiplication

$$
(f ; \bar{g})=\iint_{\Delta} f(z) \overline{g(z)} d \omega, \quad d \omega=d x d y, z=x+i y .
$$

Let $h^{2}(\Delta)$ be the class of functions which belong to the orthogonal complement of $\left[l^{2}(\Delta)\right]$ with respect to $\left[L^{2}(\Delta)\right]$. The Hilbert space $\left[h^{2}(\Delta)\right]$ has a finite dimension $p-1$, if $\Delta$ is $p$-connected and none of its boundary components reduces to a point. (See Bergman [3]). If $\Delta$ is infinitelyconnected, this space has in general an infinite dimension (See Virtanen [9]).

According to Virtanen [9] (See also Nevanlinna [7], one can construct an orthogonal basis for $h^{2}(\Delta)$ as follows: Let $C_{1}, C_{2}, \cdots$ be a homology basis of cycles in $\Delta$, subject therefore to the following conditions:

(1) Any cycle in $\Delta$ is homologous to a finite chain of these cycles.

(2) No chain is homologous to zero, unless its coefficients are all zero. We can also assume that each cycle $C_{j},(j=1,2, \cdots)^{1}$ is an oriented analytic Jordan curve. If $K_{\Delta}(z, \zeta)$ is the Bergman kernel function for the class $L^{2}(\Delta)$, then the functions

$$
F_{j}^{\prime}(z ; \Delta)=i \oint_{\sigma_{j}} \overline{K_{\Delta}(\zeta, z)} \overline{d \zeta}, \quad j=1,2, \cdots
$$

Received July 19, 1961.

1 Throughout this paper the notation $1,2, \cdots$ will mean a finite or infinite sequence, as the case may be. 
span the space $\left[h^{2}(\Delta)\right]$; and if a subsequence $F_{j_{k}}^{\prime}(z ; \Delta)$ of them is obtained by omitting those functions which depend on the previous ones, we can orthonormalize its elements and thus obtain a basis $\varphi_{n}(z ; \Delta)(n=1,2, \cdots)$ for $\left[h^{2}(\Delta)\right]$. The coeficients used in this process are real:

$$
\varphi_{n}(z ; \Delta)=\sum_{k=1}^{n} c_{k}^{(n)} F_{j_{k}}^{\prime}(z ; \Delta) ; \quad c_{k} \text { real, } n=1,2, \cdots .
$$

We recall also that

$$
F_{j}^{\prime}(z ; \Delta)=2 \frac{\partial}{\partial z} \omega_{j}(z ; \Delta), \quad 2 \frac{\partial}{\partial z}=\frac{\partial}{\partial x}-i \frac{\partial}{\partial y},
$$

where $\omega_{j}(z ; \Delta)$ are the harmonic measures of that part of the boundary of $\Delta$, which lies inside the cycle $C_{j}$. (See Bergman [3], Virtanen [9]).

Clearly, the classes $L^{2}(\Delta)$ and $l^{2}(\Delta)$ decrease monotonically if the domain $\Delta$ increases, and this fact plays an important role in the development of the theory of the kernel function. Unfortunately, a monotonic property for the class $h^{2}(\Delta)$ does not hold in general. It is therefore interesting to notice that if $B$ is a symmetric domain with respect to the real axis, and the extended real axis is contained in $B$, then

$$
h^{2}(D) \subset h^{2}(B),
$$

where $D$ is that part of $B$ which lies in the upper part of the finite plane. (Section 2). We study the connection between the bases for the classes $h^{2}(B)$ and $h^{2}(D)$, and deduce various relations for the kernel functions and the $l$-kernels for the corresponding classes. (Sections 2, 3).

2. Symmetric domains. Let $D$ be a finitely or infinitely-connected domain in the $z$-plane, the boundary of which consists of the real axis $I$ as well as other boundary components which shall be denoted by $\Gamma$. We assume that $I$ is an isolated boundary component and we denote by $D^{*}$ and $\Gamma^{*}$ the reflections of $D$ and $\Gamma$ with respect to $I$, respectively. Let $B$ be the symmetric domain bounded by $\Gamma$ and $\Gamma^{*}$.

Let $C_{j}(j=1,2, \cdots)$ be a basis for the homology group of $D$, as described in $\S 1$. We choose the orientation on $C_{j}$ to be counter clockwise and therefore $I$ is not in the interior of $C_{j}(j=1,2, \cdots)$. We also assume that $C_{1}$ encloses $\Gamma$. Let $C_{j}^{*}(j=1,2, \cdots)$ be the reflection of $C_{j}$ with respect to $I$, oriented counter clockwise. Clearly, the $C_{j}^{*}$ 's form a homology basis for $D^{*}$, and $C_{1}, C_{2}, \cdots, C_{2}^{*}, C_{3}^{*}, \cdots$ form a homology basis for $B$. We also denote by $\Gamma_{j}, \Gamma_{j}^{*}$ those parts of $\Gamma$ and $\Gamma^{*}$ which are enclosed by $C_{j}$ and $C_{j}^{*}$, respectively $(j=1,2, \cdots) .\left(\Gamma \equiv \Gamma_{1}\right)$.

The harmonic measures $\omega_{j}(z ; D)$ of $\Gamma_{j}$, with respect to $D$, vanish on $I$ and therefore can be continued into $D^{*}$. They satisfy: 


$$
\omega_{j}(z ; D)=-\omega_{j}(\bar{z} ; D)
$$$$
z \in B, j=1,2, \cdots \text {. }
$$

Thus, the functions

$$
F_{j}^{\prime}(z ; D) \equiv 2 \frac{\partial \omega_{j}(z ; D)}{\partial z}, \quad j=1,2, \cdots
$$

are single-valued and regular throughtout $B$, and satisfy

$$
F_{j}^{\prime}(z ; D)=\overline{-F_{j}^{\prime}(\bar{z} ; D)}, \quad j=1,2, \cdots, z \in B .
$$

Here and elsewhere we use the notation

$$
F_{j}^{\prime}(\bar{z} ; D)=\left.\frac{d F_{j}(\zeta ; D)}{d \zeta}\right|_{\zeta=\bar{z}}
$$

By (1.3) it now follows that

$$
\varphi_{n}(z ; D)=\overline{-\varphi_{n}(\bar{z} ; D)}, \quad z \in B, n=1,2, \cdots .
$$

THEOREM 2.1. If $B$ is a symmetric domain with respect to the real axis, and contains the real axis, then

$$
h^{2}(D) \subset h^{2}(B) \text {. }
$$

$D$ denotes that part of $B$ which lies in the upper half plane.

Proof. The functions $\varphi_{j}(z ; D) / \sqrt{2}(j=1,2, \cdots)$ belong to the class $h^{2}(B)$ and form an orthonormal system in $B$. Indeed, if $g(z) \in l^{2}(B)$ then a fortiori $g(z) \in l^{2}(D)$ and $\overline{g(\bar{z})} \in l^{2}(D), z \in D$; hence, by (2.5),

$$
\iint_{B} \varphi_{n}(z ; D) \overline{g(z)} d \omega=\iint_{D} \varphi_{n}(z ; D) \overline{g(z)} d \omega-\iint_{D} \overline{\varphi_{n}(z ; D) g(\bar{z})} d \omega=0 .
$$

Similarly,

$$
\begin{aligned}
& \iint_{B} \varphi_{n}(z ; D) \overline{\varphi_{m}(z ; D)} d \omega=\iint_{D} \varphi_{n}(z ; D) \overline{\varphi_{m}(z ; D)} d \omega \\
& \quad+\iint_{D} \overline{\varphi_{n}(z ; D)} \varphi_{m}(z ; D) d \omega=2 \delta_{n m} .
\end{aligned}
$$

If $f(z) \in h^{2}(D)$, then $f(z)=\Sigma_{n} a_{n} \varphi_{n}(z ; D)$, where $\Sigma_{n}\left|a_{n}\right|^{2}<\infty$. Thus $\Sigma_{n}\left|\sqrt{2} a_{n}\right|^{2}<\infty$; hence $f(z)=\Sigma_{n} \sqrt{2} a_{n}\left(\varphi_{n}(z) / \sqrt{2}\right) \in h^{2}(B)$.

Consider again the symmetric domain $B$, and let $\omega_{j}(z ; B), \omega_{j}^{*}(z ; B)$ be the harmonic measures of $\Gamma_{j}, \Gamma_{j}^{*}$, with respect to $B$, respectively. We shall prove that

$$
\omega_{j}^{*}(z ; B)=\omega_{j}(\bar{z} ; B), \quad z \in B, j=1,2, \cdots
$$


Indeed, we exhaust $B$ by a sequence of finitely-connected subdomains $B_{1}, B_{2}, \cdots$,

$$
\bar{B}_{m} \subset B_{m+1}, \bigcup_{m} B_{m}=B, \quad m=1,2, \cdots .
$$

We can assume that each domain is bounded by analytic Jordan curves and is symmetric and contains the extended real axis. Clearly, for each $j$,

$$
\omega_{j}^{*}\left(z ; B_{m}\right)=\omega_{j}\left(\bar{z} ; B_{m}\right),
$$

if $m$ is large enough, since both sides are harmonic in $B_{m}$ and have the same boundary values. By letting $m$ go to infinity, we obtain (2.9). (See Virtanen [9]).

In a similar fashion one obtains:

$$
\omega_{j}(z ; B)-\omega_{j}(\bar{z} ; B)=\omega_{j}(z ; D), z \in B, \quad j=1,2, \cdots .
$$

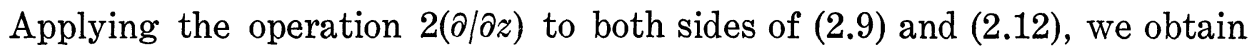
the important relations:

$$
\begin{aligned}
F_{j}^{\prime *}(z ; B) & =\overline{F_{j}^{\prime}(\bar{z} ; B)} \\
\left.F_{j}^{\prime}(z ; B)-\overline{F_{j}^{\prime}(\bar{z} ; B}\right) & =F_{j}^{\prime}(z ; D), \quad z \in B, j=1,2, \cdots .
\end{aligned}
$$

Since $h^{2}(D) \subset h^{2}(B)$, it is of interest to have some information on the orthogonal complement of $\left[h^{2}(D)\right]$ with respect to $\left[h^{2}(B)\right]$.

THEOREM 2.2. Let $B$ be a symmetric domain with respect to the real axis, which contains the real axis, and let $D$ be that part of $B$ which lies in the upper half plane. Let ${ }^{2}$

$$
\begin{gathered}
\psi_{n}(z ; B)=\varphi_{n}(z ; D) / \sqrt{2}=\sum_{k=1}^{n} c_{n k} F_{k}^{\prime}(z ; D),\left(c_{n k} \text { real }\right), \\
\chi_{n}(z ; B)=\sum_{k=1}^{n} c_{n k} F_{k}^{\prime}(z ; B),
\end{gathered}
$$

where $\varphi_{n}(z ; D)$ is an orthonormal basis for $\left[h^{2}(D)\right]$, as constructed in $\S 1$. Then, the functions

$$
r_{n}(z ; B)=\chi_{n}(z ; B)-\frac{\psi_{n}(z ; B)}{2}
$$

are orthogonal to the functions

$$
\psi_{m}(z ; B) \quad n, m=1,2, \cdots .
$$

Proof. It is well known (see Bergman [3], Virtanen [9]) that the

${ }^{2}$ We assume that $F_{k}^{\prime}(z ; D), k=1,2, \cdots$, are already independent. Note also that $F_{1}^{\prime}(z ; D)=2 F_{1}^{\prime}(z ; B)$ (see Section 1$)$. 
scalar product (over $B$ ) of any two functions from the closed system $\left\{F_{j}^{\prime}(z ; D), F_{j+1}^{\prime}(z ; B), j=1,2, \cdots\right\}$ is real. The same holds true for the scalar product of $\psi_{m}(z ; B)$ and $\chi_{n}(z ; B)$. Therefore, in view of (2.5) and (2.14),

$$
\begin{aligned}
& \iint_{B} \chi_{n}(z ; B) \overline{\psi_{m}(z ; B)} d \omega \\
= & \left.\iint_{D} \chi_{n}(z ; B) \overline{\psi_{m}(z ; B}\right) d \omega-\iint_{D *} \chi_{n}(z ; B) \psi_{m}(\bar{z} ; B) d \omega \\
= & \iint_{D}\left(\psi_{n}(z ; B)+\overline{\chi_{n}(\bar{z} ; B)}\right) \overline{\psi_{m}(z ; B)} d \omega-\iint_{D} \chi_{n}(\bar{z} ; B) \psi_{m}(z ; B) d \omega \\
= & \iint_{D} \psi_{n}(z ; B) \overline{\psi_{m}(z ; B)} d \omega=\frac{1}{2} \delta_{n m},
\end{aligned}
$$

where $\delta_{n m}=0$ if $n \neq m, \delta_{n n}=1$.

On the other hand,

$$
\iint_{B} \frac{\psi_{n}(z ; B)}{2} \overline{\psi_{m}(z ; B)} d \omega=\frac{1}{2} \delta_{n m} ;
$$

hence the result follows.

COROLlaRY 2.1. It is easily verified that

$$
r_{n}(z ; B)=\overline{r_{n}(\bar{z} ; B),} \quad r_{1}(z ; B)=0 ；
$$

hence, by orthonormalizing the functions $r_{n}(z ; B), n=2,3, \cdots$ (with real coefficients), a complete orthonormal basis $\psi_{n}(z ; B), \rho_{n}(z ; B), n=1,2, \cdots$, is constructed for $\left[h^{2}(B)\right]$, where

$$
\psi_{n}(z ; B)=-\overline{\psi_{n}(\bar{z} ; B)}, \rho_{n}(z ; B)=\overline{\rho_{n}(\bar{z} ; B)}, \quad n=1,2, \cdots .
$$

This result, which later will be of much use, implies:

THEOREM 2.3. Let $B$ be a symmetric domain with respect to the real axis, and contains the real axis, then, each function $f(z)$ of the class $h^{2}(B)$ can be represented as a sum of two functions $f_{1}(z)$ and $f_{2}(z)$, of the same class, which satisfy

$$
f_{1}(z)=-\overline{f_{1}(\bar{z})}, \quad f_{2}(z)=\overline{f_{2}(\bar{z})} .
$$

In fact,

$$
2 f_{1}(z)=f(z)-\overline{f(\bar{z})}, \quad 2 f_{2}(z)=f(z)+\overline{f(\bar{z})} .
$$

3. The space $h_{r}^{2}(\Delta)$. Let $h_{r}^{2}(\Delta)$ be a subclass of $h^{2}(\Delta)$, consisting of those functions which have real Fourier coefficients with respect to the base $\varphi_{n}(z ; \Delta)(n=1,2, \cdots)$. This class forms a real Hilbert space $\left[h_{r}^{2}(\Delta)\right]$, 
with respect to the metric (1.1).

In order to extend some results to the case of a general domain 4 , we exhaust $\Delta$ by a sequence of smoothly bounded, finitely-connected subdomains $\left\{A_{m}\right\}, m=1,2, \cdots$ which satisfy

$$
\bar{\Delta}_{m} \subset \Delta_{m+1}, \bigcup_{m} \Delta_{m}=\Delta,
$$$$
m=1,2, \cdots
$$

LEMmA 3.1. If $f(z) \in h_{r}^{2}(\Delta)$, there exists a sequence of functions $\left\{f_{m}(z)\right\}, f_{m}(z) \in h_{r}^{2}\left(\Delta_{m}\right), m=1,2, \cdots$, which converges in the mean to $f(z)$, and therefore converges uniformly to $f(z)$ on each compact set in $\Delta$.

Proof. Given $f(z)$, it follows that

$$
f(z)=\Sigma_{n} a_{n} \rho_{n}(z ; \Delta), \quad a_{n} \text { real, } \Sigma_{n} a_{n}^{2}<\infty .
$$

The series converges in the mean. If $m$ is large enough, $\Delta_{m}$ contains a given cycle $C_{j}$ which encloses a part $\Gamma_{i}^{(m)}$ of the boundary of $A_{m}$. It is known (see Virtanen [9]) that $F_{j}^{\prime}\left(z ; \Delta_{m}\right)$ converges in the mean to $F_{j}^{\prime}(z, \Delta)$ if $m \rightarrow \infty$ (we define $F_{j}^{\prime}\left(z ; \Delta_{m}\right)$ to be zero outside $\Delta_{m}$ ). One now applies the Gram-Schmidt orthonormalization process to a subsequence $\left\{F_{j_{k}}^{\prime}\left(z ; \Delta_{m}\right)\right\}$, where $k$ is chosen in such a way that each $F_{j_{k}}^{\prime}(z ; \Delta)$ is independent on the functions $F_{j_{l}}^{\prime}(z ; \Delta), l=1,2, \cdots, k-1$. One obtains for each $m$ an orthonormal system

$$
\varphi_{n}\left(z, \Delta_{m}\right)=\sum_{k=1}^{N_{m}} c_{k}^{(m)} F_{j_{k}}^{\prime}\left(z ; \Delta_{m}\right), \quad c_{k}^{(m)} \text { real, } k=1,2, \cdots, N_{m} .
$$

$N_{m}$ is the number of cycles $C_{j_{k}}$ which are in $A_{m}$. It can be proved by induction that $\varphi_{n}\left(z ; \Delta_{m}\right)$ converges in the mean to $\varphi_{n}(z ; \Delta)$, defined by (1.3) and that $c_{k}^{(m)} \rightarrow c_{k}$, as $m \rightarrow \infty$. Consequently, the functions

$$
f_{m}(z)=\sum_{n=1}^{N m} a_{n} \varphi_{n}\left(z ; \Delta_{m}\right)
$$

converge to $f(z)$ in the mean.

The space $h_{r}^{2}(\Delta)$ can be characterized differently. To this effect we apply the $l$-kernel defined (Bergman Schiffer [4], Schiffer [5]) as

$$
l_{\Delta}(z, \zeta)=\frac{1}{\pi(z-\zeta)^{2}}+\frac{2}{\pi} \frac{\partial^{2} G_{\Delta}(z, \zeta)}{\partial z \partial \zeta},
$$

where $G_{\Delta}(z, \zeta)$ is the Green function of the domain $\Delta$.

LEMma 3.2. The kernels $l_{\Delta_{m}}(z, \zeta)$ for a fixed $\zeta$, converges in the mean to $l_{\Delta}(z, \zeta) . \quad \Delta_{m}$ is the exhaustion (3.1).

Proof. We use the known relations (Bergman-Schiffer [4]) 


$$
\iint_{\Delta_{m}} l_{\Delta_{m}}(z, \zeta) \overline{l_{\Delta_{m}}(z, \zeta)} d \omega_{z}=K_{\Delta_{m}}(\zeta, \zeta)-\Gamma_{\Delta_{m}}(\zeta, \zeta)
$$

where

$$
\Gamma_{\Delta_{m}}(\zeta, \zeta)=\frac{1}{\pi^{2}} \iint_{\tilde{a}_{m}} \frac{d \omega_{z}}{|z-\zeta|^{4}}, \quad \zeta \in \Delta_{m}
$$

$\widetilde{\Delta}_{m}$ is the complement of $\Delta_{m}$ with respect to the $z$-plane.

$$
T_{\Delta_{m}} f \equiv \iint_{\Delta_{m}} l(z, \zeta) \overline{f(z)} d \omega_{z}=\frac{1}{\pi} \iint_{\Delta_{m}} \frac{\overline{f(z)}}{(z-\zeta)^{2}} d \omega_{z}, \zeta \in \Delta,
$$

for $f(z) \in L^{2}\left(\Delta_{m}\right)$. The improper integral on the right hand side of (3.8) is to be understood in the sense of the limit of integrals over the domains $\Delta_{\varepsilon, m}$ which are obtained from $\Delta_{m}$ by removing circles around $\zeta$ with radius $\varepsilon, \varepsilon \rightarrow 0$. Let $p \geqq m$, then

$$
\begin{aligned}
& \iint_{\Delta_{m}}\left|l_{\Delta_{p}}(z, \zeta)-l_{\Delta_{m}}(z, \zeta)\right|^{2} d \omega_{z} \leqq K_{\Delta_{p}}(\zeta, \zeta)-\Gamma_{\Delta_{p}}(\zeta, \zeta) \\
& \quad+K_{\Delta_{m}}(\zeta, \zeta)-\Gamma_{\Delta_{m}}(\zeta, \zeta)-2 \operatorname{Re} \iint_{\Delta_{m}} l_{\Delta_{p}}(z, \zeta) \overline{l_{\Delta_{m}}(z, \zeta)} d \omega_{z} .
\end{aligned}
$$

By (3.8), the last integral can be estimated:

$$
\begin{aligned}
& \iint_{\Delta_{m}} l_{\Delta_{p}}(z, \zeta) \overline{l_{\Delta_{m}}(z, \zeta)} d \omega_{z}=\frac{1}{\pi} \iint_{\Delta_{m}} \frac{l_{\Delta_{p}}(z, \zeta)}{(\bar{z}-\bar{\zeta})^{2}} d \omega_{z} \\
= & \iint_{\Delta_{p}} l_{\Delta_{p}}(z, \zeta) \overline{l_{\Delta_{p}}(z, \zeta)} d \omega_{z}-\frac{1}{\pi} \iint_{\Delta_{p}-\Delta_{m}} \frac{l_{\Delta_{p}}(z, \zeta)}{(\bar{z}-\bar{\zeta})^{2}} d \omega_{z} \\
= & K_{\Delta_{p}}(\zeta, \zeta)-\Gamma_{\Delta_{p}}(\zeta, \zeta)-\frac{1}{\pi} \iint_{\Delta_{p-\Delta_{m}}} \frac{l_{\Delta_{p}}(z, \zeta)}{(\bar{z}-\bar{\zeta})^{2}} d \omega_{z} .
\end{aligned}
$$

By Schwarz inequality, the modulus of the last integral can be made arbitrarily small, if $m$ is large enough, and also $K_{\Delta_{m}}(\zeta, \zeta)$ tends to $K_{\Delta}(\zeta, \zeta)$ (See Virtanen [9]); hence the right hand side of (3.9) tends to zero if $m \rightarrow \infty$.

The limit function is $l_{\Delta}(z, \zeta)$ as defined by 3.5 , since $G_{\Delta_{m}}(z, \zeta)$ converges to $G_{\Delta}(z, \zeta)$.

Theorem 3.1. (Bergman-Schiffer) The function $f(z) \in h^{2}(\Delta)$ belongs to the class $h_{r}^{2}(\Delta)$ if and only if

$$
T_{\Delta} f \equiv \iint_{\Delta} l_{\Delta}(z, \zeta) \overline{f(z)} d \omega_{z}=-f(\zeta),
$$

This theorem was proved in [4] for the case of a sufficiently smooth bounded, finitely-connected domain. If $f(z) \in h_{r}^{2}(\Delta)$, where $\Delta$ is a general 
domain, it follows from Lemma 3.1 and Lemma 3.2 that $T_{\Delta_{m}} f_{m}(z)=-f_{m}(\zeta)$ implies $T_{\Delta} f(z)=-f(\zeta)$. Conversly, if $f(z) \in h^{2}(\Delta)$ then

$$
f(z)=\Sigma \alpha_{n} \varphi_{n}(z ; \Delta)+i \Sigma \beta_{n} \varphi_{n}(z ; \Delta), \alpha_{n}, \beta_{n} \text { real ; }
$$

hence $T_{\Delta} f(z)=-f(z)$ can only happen if $\beta_{n}=0$.

If is known [4] that $l_{\Delta}(z, \zeta)$ can be represented as a sum tof two kernels:

$$
l_{\Delta}(z, \zeta)=l_{\Delta}^{(l)}(z, \zeta)+l_{\Delta}^{(h)}(z, \zeta)
$$

where ${ }^{3}$

$$
l_{\Delta}^{(h)}(z, \zeta)=-\sum_{n} \varphi_{n}(z, \Delta) \varphi_{n}(\zeta, \Delta),
$$

and $l_{\Delta}^{(l)}(z, \zeta)$ belongs to $l^{2}(\Delta)$, for each fixed $\zeta$. We therefore obtain:

Corollary. A necessary and sufficient condition that a function $f(z)$ of the class $L^{2}(\Delta)$ belongs to $h_{r}^{2}(\Delta)$ is

$$
T_{\Delta}^{(r)} \equiv \iint_{\Delta} l_{\Delta}^{(h)}(z, \zeta) \overline{f(z)} d \omega_{z}=-f(\zeta)
$$

REMARK. Clearly, $K_{\Delta}^{(k)}(z, \zeta)-l_{\Delta}^{(h)}(z, \zeta) \in h_{r}^{2}(\Delta)$ as a function of $z$, where

$$
K_{\Delta}^{(h)}(z, \zeta)=\sum_{n} \varphi_{n}(z ; \Delta) \overline{\varphi_{n}(\zeta ; \Delta)} .
$$

In the case of a symmetric domain $B$, Theorem 2.1 can be slightly improved to the effect that $h_{r}^{2}(D) \subset h_{r}^{2}(B)$. Also, if $f(z) \in h_{r}^{2}(D)$, it follows from (2.5) that

$$
f(z)=-\overline{f(\bar{z})} .
$$

Limiting ourselves to the spaces $h_{r}^{2}(B), h_{r}^{2}(D)$, we can now construct functions in the latter space from functions in the former one:

THEOREM 3.2. If $B$ is a symmetric domain with respect to the real axis, and contains the real axis, and if $f(z) \in h_{r}^{2}(B)$ then $f(z)-\overline{f(\bar{z})} \in h_{r}^{2}(D)$, where $D$ is that part of $B$ which lies in the upper half plane.

Proof. We exhaust $B$ by the symmetric domains $\left\{B_{m}\right\}$ (See (2.10)), and consider the functions $f_{m}(z)$, defined in (3.4), where $f(z)$ has the representation (3.2). The functions $\varphi_{n}\left(z ; B_{m}\right)$ are finite linear combinations, with real coefficients, of $F_{j_{k}}^{\prime}\left(z ; B_{m}\right)$ and $F_{j_{k}}^{* \prime}\left(z ; B_{m}\right)$; consequently, by (2.13) and (2.14), $\varphi_{n}\left(z ; B_{m}\right)-\varphi_{n}\left(\overline{\bar{z} ; B_{m}}\right)$ are finite linear combintations, with real coefficients, of $F_{j_{k}}^{\prime}\left(D_{m}\right)$, which, in turn, are finite linear combintations,

${ }^{3}$ The generalization to a general domain is obvious. 
with real coefficients, of $\varphi_{n}\left(z ; D_{m}\right)$. Since $\left[h_{r}^{2}\left(\Delta_{m}\right)\right]$ has finite dimensions, it follows that

$$
f_{m}(z)-\overline{f_{m}(\bar{z})} \equiv g_{m}(z) \in h_{r}^{2}\left(D_{m}\right) .
$$

Thus, by Lemma 3.1 if $m \rightarrow \infty$, the left hand side converges to the function $f(z)-\overline{f(\bar{z})}=g(z)$. But $g(z) \in L^{2}(B) \subset L^{2}(D)$, hence $g_{m}(z)$ tends to $g(z)$ which is in $h_{r}^{2}(D)$.

This theorem could also be proved directly by the use of Corollary 2.1. We can also use this corollary and the series developments (3.14), (3.16) in order to prove by direct calculation the following

THEOREM 3.3. If $B$ is a symmetric domain with respect to the real axis, and contains the real axis, then the following identities hold for $z, \zeta \in B$.

$$
\begin{gathered}
K_{B}^{(h)}(z, \zeta)=K_{B}^{(h)}(\bar{\zeta}, \bar{z}), \\
l_{B}^{(h)}(z, \zeta)=\overline{l_{B}^{(h)}(\bar{z}, \bar{\zeta})}, \\
K_{B}^{(h)}(z, \bar{\zeta})+l_{B}^{(h)}(z, \zeta)=K_{D}^{(h)}(z, \bar{\zeta}), \\
K_{D}^{(h)}(z, \bar{\zeta})=\overline{K_{D}^{(h)}(\bar{\zeta}, z)}=l_{D}^{(h)}(z, \zeta) .
\end{gathered}
$$

Here $D$ denotes that part of $B$, which lies in the upper half plane.

REMARK. From the symmerty of $B$, it follows that the Green function satisfies the identity

$$
G_{B}(z, \zeta)=G_{B}(\bar{z}, \bar{\zeta}),
$$$$
z, \zeta \in B
$$

therefore, by (3.5) and by the well known formula (See [4], [5])

$$
K_{\Delta}(z, \zeta)=-\frac{2}{\pi} \frac{\partial^{2} G_{\Delta}(z, \zeta)}{\partial z \partial \bar{\zeta}}
$$

it follows also that

$$
\begin{aligned}
& K_{B}(z, \zeta)=K_{B}(\bar{\zeta}, \bar{z}) \\
& l_{B}(z, \zeta)=\overline{l_{B}(\bar{z}, \bar{\zeta})} .
\end{aligned}
$$

Hence, similar identities hold also for the kernels $K_{B}^{l}(z, \zeta)$ and $l_{B}^{l}(z, \zeta)$. Making use of Corollary 2.1 (2.15), (3.14), (3.16), we also find that

$$
K_{B}^{(h)}(z, \zeta)-\frac{1}{2} K_{D}^{(h)}(z, \zeta)=\frac{1}{2} l_{D}^{(h)}(z, \bar{\zeta})-l_{B}^{(h)}(z, \bar{\zeta})=\sum_{n} \rho_{n}(z) \overline{\rho_{n}(\zeta)}
$$

hence,

THEOREM 3.4. If $B$ is a symmetric domain with respect to the real 
axis $I$, and contains the real axis, and if $D$ is that part of $B$ which lies in the upper half plane, then

$$
\begin{aligned}
K_{B}^{(h)}(z, z) \geqq \frac{1}{2} K_{D}^{(h)}(z, z), & & z \in B, z \notin I . \\
l_{B}^{(h)}(z, \bar{z}) \leqq \frac{1}{2} l_{D}^{(h)}(z, \bar{z}), & & z \in B, z \notin I .
\end{aligned}
$$

4. Concluding remarks. A domain $\Delta$ is said to be of zero span (of the class $0_{A D}$, in the terminology of the theory of Riemann Surfaces), if the class $l^{2}(\Delta)$ reduces to the zero function. (See Ahlfors-Sario [2], Nevanlinna [7], Schiffer [8]). This property is invariant under univalent conformal mappings, and the boundary of $\Delta$ can be characterized by the fact that it is a removable singularity for all the functions, defined in a neighbourhood of the boundary and belong to the class $l^{2}$ in that neighbourhood. (See Painlevé Problem, [7]).

Clearly, for such domains,

$$
K_{\Delta}(z, \zeta)=K_{\Delta}^{(h)}(z, \zeta) \quad l(z, \zeta)=l^{(h)}(z, \zeta)
$$

Therefore, if $B$ is a symmetric domain of zero span, one can replace the class $h^{2}(B)$ by $L^{2}(B)$ in all our previous results. One may also deduce properties of the class $h^{2}(D)$ from known properties of the class $L^{2}(B)$.

In Maschler [6], it was conjectured that if there exists a point in a domain $\Delta$, at which all the functions of the class $h^{2}(\Delta)$ vanish, then $h^{2}(\Delta)$ consists of the zero function alone. (In the later case, the domain is simply-connected except for punctures which are removable with respect to the function of $h^{2}(\Delta)$ ). This conjecture was proved there under the assumption that $\Delta$ has two boundary components which are not completely point-like. An analogous statement is correct for the classes $L^{2}(\Delta)$ (see Nevanlinna [7]) and $l^{2}(\Delta)$ (see Ahlfors Beurling [1]). ${ }^{4}$ The above mentioned conjecture is trivially true if $\Delta$ has a zero span.

Using the representation (2.21), one derives easily, by (2.15), that there exists a point $t$ in $D$, at which all the functions of the class $h^{2}(D)$ vanish, if and only if $f(t)=\overline{f(\bar{t})}$ for all the functions $f(z)$ of the class $h_{r}^{2}(B)$. Thus, if $\Gamma$ contains a boundary component which is not completely point-like, then, for any point $t$ in $D$, there exists a function of the class $h_{r}^{2}(B)$, which does not take a conjugate value at $\bar{t}$.

It would be of interest to study also the question whether it is possible that all the functions $f(z)$ of the class $h_{r}^{2}(B)$ satisfy at a particular point $t, t \in D$, the relation $f(t)=-\overline{f(\bar{t})}$. This is equivalent to equality in (3.28) and (3.29), for $z=t$. (See (3.27)).

${ }_{4}$ The situation in the general case of a Riemann surface is studied in Virtanen [10]. 


\section{REFERENCES}

1. L. V. Ahlfors and A. Beurling, Conformal invariants and function-theoretic null-sets. Acta Math., 83 (1950), 101-129.

2. L. V. Ahlfors and L. Sario, Riemann Surfaces, Princeton Univ. Press, Princeton New Jersey (1960).

3. S. Bergman, The kernel function and conformal mapping. Math. Surveys V, Amer. Math. Soc., New York (1950).

4. S. Bergman and M. Schiffer, Kernel functions and conformal mapping, Composition Math., 8 (1950), 205-249.

5. R. Courant, Dirichlet's principle, Conformal mapping and minimal surfaces, Appendix by M. Schiffer. Interscience pub. New York (1950).

6. M. Maschler, Analytic functions of the classes $L^{2}$ and $l^{2}$ and their kernel functions, Rendiconti Circ. Mat. Palermo, Ser. II, 8 (1959), 163-177.

7. R. Nevanlinna, Uniformisierung, Springer-Verlag, Berlin (1953).

8. M. Schiffer, The span of multiply-connected domains, Duke Math. J., 10 (1943), 209-216.

9. K. I. Virtanen, Über eine Integraldarstellung von quadratische integrierbaren analytischen Differentialen, Ann. Acad. Scient. Fenn. Ser. A, I, 69 (1950).

10. K. I. Virtanen, Über Extremalfunktionen auf offenen Riemannschen Flächen, Ann.

Acad. Scient. Fenn. Ser. A, I, 141 (1952). 



\title{
PACIFIC JOURNAL OF MATHEMATICS
}

\author{
EDITORS
}

Ralph S. Phillips

Stanford University

Stanford, California

M. G. Arsove

University of Washington

Seattle 5, Washington
A. L. Whiteman

University of Southern California Los Angeles 7, California

Lowell J. Paige

University of California

Los Angeles 24, California

\section{ASSOCIATE EDITORS}
E. F. BECKENBACH
D. DERRY
M. OHTSUKA
H. L. ROYDEN
E. SPANIER
E. G. STRAUS
T. M. CHERRY
F. WOLF

\section{SUPPORTING INSTITUTIONS}

\author{
UNIVERSITY OF BRITISH COLUMBIA \\ CALIFORNIA INSTITUTE OF TECHNOLOGY \\ UNIVERSITY OF CALIFORNIA \\ MONTANA STATE UNIVERSITY \\ UNIVERSITY OF NEVADA \\ NEW MEXICO STATE UNIVERSITY \\ OREGON STATE UNIVERSITY \\ UNIVERSITY OF OREGON \\ OSAKA UNIVERSITY \\ UNIVERSITY OF SOUTHERN CALIFORNIA
}

\author{
STANFORD UNIVERSITY \\ UNIVERSITY OF TOKYO \\ UNIVERSITY OF UTAH \\ WASHINGTON STATE UNIVERSITY \\ UNIVERSITY OF WASHINGTON \\ $*$
AMERICAN MATHEMATICAL SOCIETY \\ CALIFORNIA RESEARCH CORPORATION \\ SPACE TECHNOLOGY LABORATORIES \\ NAVAL ORDNANCE TEST STATION
}

Mathematical papers intended for publication in the Pacific Journal of Mathematics should be typewritten (double spaced), and the author should keep a complete copy. Manuscripts may be sent to any one of the four editors. All other communications to the editors should be addressed to the managing editor, L. J. Paige at the University of California, Los Angeles 24, California.

50 reprints per author of each article are furnished free of charge; additional copies may be obtained at cost in multiples of 50 .

The Pacific Journal of Mathematics is published quarterly, in March, June, September, and December. Effective with Volume 13 the price per volume (4 numbers) is $\$ 18.00$; single issues, $\$ 5.00$. Special price for current issues to individual faculty members of supporting institutions and to individual members of the American Mathematical Society: $\$ 8.00$ per volume; single issues $\$ 2.50$. Back numbers are available.

Subscriptions, orders for back numbers, and changes of address should be sent to Pacific Journal of Mathematics, 103 Highland Boulevard, Berkeley 8, California.

Printed at Kokusai Bunken Insatsusha (International Academic Printing Co., Ltd.), No. 6, 2-chome, Fujimi-cho, Chiyoda-ku, Tokyo, Japan.

PUBLISHED BY PACIFIC JOURNAL OF MATHEMATICS, A NON-PROFIT CORPORATION

The Supporting Institutions listed above contribute to the cost of publication of this Journal, but they are not owners or publishers and have no responsibility for its content or policies. 


\section{Pacific Journal of Mathematics}

\section{Vol. 12, No. $2 \quad$ February, 1962}

William George Bade and Robert S. Freeman, Closed extensions of the Laplace operator determined by a general class of boundary conditions . . . . . . . 395

William Browder and Edwin Spanier, H-spaces and duality ............. 411

Stewart S. Cairns, On permutations induced by linear value functions . . . . . . . 415

Frank Sydney Cater, On Hilbert space operators and operator roots of

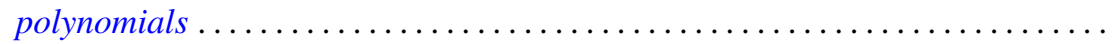

Stephen Urban Chase, Torsion-free modules over $K[x, y] \ldots \ldots \ldots \ldots \ldots \ldots \ldots 437$

Heron S. Collins, Remarks on affine semigroups . . . . . . . . . . . . . . . . 449

Peter Crawley, Direct decompositions with finite dimensional factors . . . . . . . 457

Richard Brian Darst, A continuity property for vector valued measurable

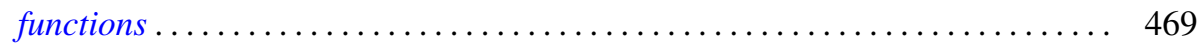

R. P. Dilworth, Abstract commutative ideal theory ................. 481

P. H. Doyle, III and John Gilbert Hocking, Continuously invertible spaces . . . . . . 499

Shaul Foguel, Markov processes with stationary measure . . . . . . . . . . . 505

Andrew Mattei Gleason, The abstract theorem of Cauchy-Weil ............ 511

Allan Brasted Gray, Jr., Normal subgroups of monomial groups . . . . . . . . . . 527

Melvin Henriksen and John Rolfe Isbell, Lattice-ordered rings and function

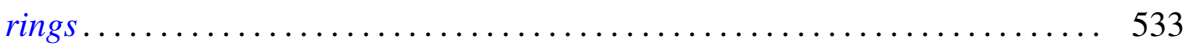

Amnon Jakimovski, Tauberian constants for the $[J, f(x)]$ transformations . ..... 567

Hubert Collings Kennedy, Group membership in semigroups . . . . . . . . . . . 577

Eleanor Killam, The spectrum and the radical in locally $m$-convex algebras ..... 581

Arthur H. Kruse, Completion of mathematical systems . . . . . . . . . . . . . 589

Magnus Lindberg, On two Tauberian remainder theorems ................ 607

Lionello A. Lombardi, A general solution of Tonelli's problem of the calculus of

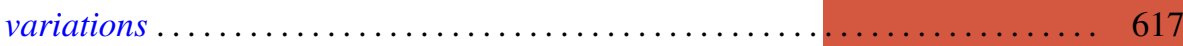

Marvin David Marcus and Morris Newman, The sum of the elements of the powers

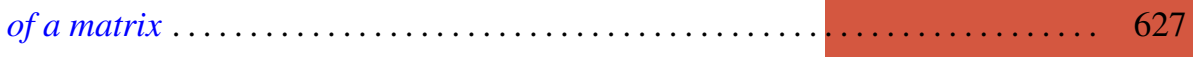

Michael Bahir Maschler, Derivatives of the harmonic measures in

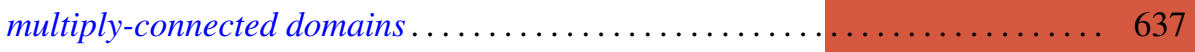

Deane Montgomery and Hans Samelson, On the action of $\mathrm{SO}(3)$ on $S^{n} \ldots \ldots \ldots 649$

J. Barros-Neto, Analytic composition kernels on Lie groups . . . . . . . . . . . . 661

Mario Petrich, Semicharacters of the Cartesian product of two semigroups ...... 679

John Sydney Pym, Idempotent measures on semigroups . . . . . . . . . . . . 685

K. Rogers and Ernst Gabor Straus, A special class of matrices . . . . . . . . . . . . 699

U. Shukla, On the projective cover of a module and related results . . . . . . . . . 709

Don Harrell Tucker, An existence theorem for a Goursat problem . . . . . . . . . . . 719

George Gustave Weill, Reproducing kernels and orthogonal kernels for analytic

differentials on Riemann surfaces ......................... 729

George Gustave Weill, Capacity differentials on open Riemann surfaces ........ 769

G. K. White, Iterations of generalized Euler functions . . . . . . . . . . . . . 777

Adil Mohamed Yaqub, On certain finite rings and ring-logics . . . . . . . . . 785 